\title{
Corela
}

Cognition, représentation, langage

HS-29 | 2019

Questions et exclamations au prisme de plusieurs approches linguistiques

\section{La phonologie des exclamations en anglais}

\section{Stephan WILHELM}

\section{(2) OpenEdition}

10 Journals

Édition électronique

URL : http://journals.openedition.org/corela/8984

DOI : $10.4000 /$ corela.8984

ISSN : 1638-573X

Éditeur

Cercle linguistique du Centre et de l'Ouest - CerLICO

Référence électronique

Stephan WILHELM, «La phonologie des exclamations en anglais », Corela [En ligne], HS-29 | 2019, mis en ligne le 09 septembre 2019, consulté le 28 octobre 2019. URL : http://journals.openedition.org/ corela/8984; DOI : 10.4000/corela.8984

Ce document a été généré automatiquement le 28 octobre 2019

\section{(c) (i) (3) (2)}

Corela - cognition, représentation, langage est mis à disposition selon les termes de la licence Creative Commons Attribution - Pas d'Utilisation Commerciale - Partage dans les Mêmes Conditions 4.0 International. 


\title{
La phonologie des exclamations en anglais
}

\author{
Stephan WILHELM
}

\section{Introduction et conventions de représentation}

\section{Questions de définitions}

1 "Exclamations are traditionally used to express the extent to which the speaker is impressed by something." C'est ainsi que Quirk et al. (2004) définissent un phénomène que d'autres auteurs (p. ex. Huddleston et Pullum 2002) décrivent comme difficile à circonscrire ${ }^{1}$.

2 Il est malaisé de décider si cette définition s'appuie sur des critères sémantiques ou pragmatiques. Il apparaît, quoi qu'il en soit, qu'elle n'est en aucun cas de nature syntaxique. Cette précision mérite d'être soulignée, car les intonationnistes ont fréquemment tenté d'établir des correspondances entre types syntaxiques d'énoncés et schémas intonatifs. Pour ce qui est de l'exclamation, on voit que les choses ne peuvent être envisagées sous cet angle.

3 Plusieurs grammairiens, au nombre desquels Quirk et al. (2004), Huddleston et Pullum (2002) et Larreya et Rivière (2002) établissent une distinction entre ce qu'ils dénomment «constructions exclamatives » (essentiellement des énoncés en WHAT et en HOW) et tous les autres types d'énoncés exclamatifs, qu'ils regroupent sous l'appellation de non-exclamative exclamations (Huddleston et Pullum 2002). Il apparaît donc que les énoncés exclamatifs ne sauraient être associés à un type unique de construction syntaxique, ce que confirme l'étendue de la gamme des structures présentes dans le corpus (voir tableau 1).

4 C'est précisément l'absence de correspondance systématique entre type syntaxique et énoncé exclamatif qui conduit Bolinger (1989) à écrire: "[I]n the broadest sense, exclamations are [...] anything at the end of which one would put an exclamation mark." On pourrait objecter que cette définition est excessivement vague, mais elle 
offre l'avantage de fournir une base simple et objective pour constituer un corpus d'exclamations (cf. Bolinger 1989)'2.

\section{Un phénomène suprasegmental}

5 Ce n'est pas au niveau segmental, c'est-à-dire au niveau du phonème (plus petite unité distinctive observée en une langue donnée dans la chaîne parlée) qu'il convient d'examiner les spécificités phonologiques des types d'énoncés. Ces spécificités ne sont pas davantage observées au niveau lexical, c'est-à-dire au niveau du mot ou des unités lexicales. Elles se manifestent au niveau suprasegmental, c'est-à-dire prosodique ${ }^{3}$. (Les éléments suprasegmentaux sont les traits que l'on observe à un niveau supérieur à celui du segment. Le plus souvent [pas toujours], ces éléments sont identifiés sur des sections de la chaîne parlée qui recouvrent plusieurs phonèmes. Ils remplissent une fonction qui se superpose à celle des traits intrinsèques de ces derniers ${ }^{4}$.)

\begin{tabular}{|l|l|l|l|}
\hline Énoncé & Type de construction & $\begin{array}{l}\text { Présence d'une } \\
\text { Interjection }\end{array}$ & $\begin{array}{l}\text { Présence d'un } \\
\text { intensifieur }\end{array}$ \\
\hline $\mathbf{1}$ & SN (N propre) / SN + SP & & \\
\hline $\mathbf{2}$ & Construction impérative & & \\
\hline $\mathbf{3}$ & Construction exclamative & Oh & What \\
\hline $\mathbf{4}$ & Construction déclarative & Oh & \\
\hline $\mathbf{5}$ & Adv. + SN & & Why \\
\hline $\mathbf{6}$ & Proposition en THAT & & At all \\
\hline $\mathbf{7}$ & Construction déclarative & Oh /How \\
\hline $\mathbf{8}$ & «Construction » optative & & So \\
\hline $\mathbf{9}$ & Construction déclarative en SO & & \\
\hline $\mathbf{1 0}$ & $\begin{array}{l}\text { Extraposition non-canonique (IT + BE + adj. + } \\
\text { construction exclamative en HOW) }\end{array}$ & & \\
\hline $\mathbf{1 1}$ & Construction déclarative & & \\
\hline
\end{tabular}

Tableau 1 : Types de constructions syntaxiques recensés dans le corpus

6 Ce qui est vrai des types d'énoncés en général l'est en particulier des énoncés exclamatifs, que Huddleston et Pullum (2002) décrivent comme prototypiquement signalés par des éléments prosodiques plutôt que par le lexique ou la syntaxe ${ }^{5}$.

7 La composante prosodique la plus souvent associée à l'exclamation est l'intonation, c'est-à-dire la variation systématique de hauteur mélodique. Cela n'a rien d'étonnant dans la mesure où, à l'instar de l'exclamation, l'intonation est souvent associée à l'expression directe de l'émotion. Bolinger (1989) écrit par exemple: "Exclamations are 
manifestations of emotional arousal" et souligne cette similarité fonctionnelle: "[I]f intonation is basically affective [...], the connection between intonation and exclamation must be both broad and deep."

$8 \mathrm{Si}$, à l'instar de Bolinger (1989, cité supra), on considère que la présence d'un point d'exclamation constitue le critère déterminant pour identifier les énoncés exclamatifs à l'écrit, il n'est pas sans intérêt de noter que Larreya et Rivière (2002) établissent une correspondance directe entre cette marque de ponctuation et «un schéma intonatif particulier » généralement employé dans les énoncés exclamatifs à l'oral ${ }^{6}$.

\section{Le système intonatif du General American}

Le cadre théorique dans lequel nous nous situons est celui de la tradition britannique. Dans le prolongement des travaux de Walker (1787), Sweet (1877) et de leurs héritiers (par exemple Jones (1947), O'Connor et Arnold (1973) ou Halliday (1967)), nous concevons les tons comme des contours mélodiques pouvant être représentés par des symboles placés avant chaque syllabe nucléaire.

variété d'anglais utilisée pour interpréter la pièce de Tennessee Williams est caractéristique du Sud des États-Unis, l'action se déroulant à la Nouvelle-Orléans. Le système intonatif de cette variété ne diffère pas a priori de celui de celui de l'accent de référence connu sous le nom de General American (GA), lui-même très semblable à celui de la RP britannique 7 . Il comprend les 7 tons suivants :

11 ` High fall (grande chute)

2 'High rise (grande montée)

3. Low fall (petite chute)

4 , Low rise (petite montée)

5 Rise-fall (ton circonflexe)

6 Fall-rise (ton creusé)

$7 \because$ Level tone (ton uni) ${ }^{8}$

12 Pour transcrire le corpus proposé, nous complèterons cet inventaire par quelques éléments additionnels, nécessaires pour faire ressortir la spécificité de l'intonation exclamative. Nous distinguerons ainsi la petite chute haute ('), dont le point de départ se situe vers le haut et le point d'arrivée vers le milieu de la tessiture, et la grande chute $(\backslash)$, dont le point de départ se situe vers le haut et le point d'arrivée vers le bas de la tessiture, c'est-à-dire vers la " ligne de base ». Nous distinguerons de même la petite montée haute ('), dont le point de départ se situe vers le milieu et le point d'arrivée vers le haut de la tessiture, de la grande montée (/), dont le point de départ se situe vers le bas et le point d'arrivée vers le haut de la tessiture. Nous reconnaîtrons également l'existence d'une chute extrême $(\backslash \backslash)$, dont le point de départ se situe à l'extrémité haute de la tessiture et le point d'arrivée près de la ligne de base.

En utilisant ces conventions de représentation, nous proposerons de notre corpus la transcription suivante :

Transcription intonative du corpus

15 (Les barres obliques délimitent les unités intonatives, les caractères gras soulignés signalent les syllabes toniques)

1 / 'Stella / 'Stella / Stella for `star /

2 / turn that over-light /off /

3 / \oh / what a convenient lo \cation / 


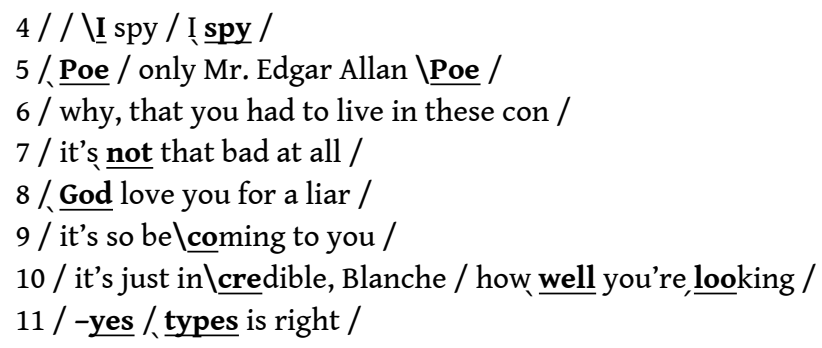

\section{Objectifs de la recherche} dans la forme des schémas intonatifs employés dans les énoncés exclamatifs. A partir de ces données, nous nous interrogerons sur les caractéristiques intonatives de l'exclamation et le fonctionnement de l'intonation dans ce type d'énoncés. Pour ce faire, nous nous pencherons tout d'abord sur la motivation de l'intonation et sur la manière dont celle-ci conditionne la forme des schémas utilisés dans les énoncés exclamatifs, puis nous examinerons les liens existant entre l'intonation des exclamations et l'expression du degré. De manière plus générale, nous nous intéresserons à la valeur démarcative de l'intonation des exclamations et à l'éclairage apporté par celle-ci sur la définition même des actes d'énonciation.

\section{La motivation de l'intonation : le cas de l'exclamation}

17 Dans son Cours de linguistique générale, Ferdinand de Saussure développe la thèse de l'arbitraire du signe linguistique. Il n'existe, selon lui, aucune motivation au lien existant entre les caractéristiques acoustiques d'un mot (le signifiant) et la représentation mentale de l'entité désignée par celles-ci (le signifié). Selon cette thèse, il n'y a par exemple pas de raison objective pour que l'image mentale de l'animal que les locuteurs de langue française connaissent sous le nom de chat $(/ \mathrm{Ja} /)$ soit représentée par cette suite de phonèmes et non par les séquences employées pour réaliser les mots souris ou encore cheval.

Notre but n'est pas d'entrer dans l'épineux débat qui s'est développé autour de la position de Saussure, mais simplement de souligner qu'il existe des cas où l'on reconnaît que le lien entre signifiant et signifié n'obéit à pas cette règle. C'est vrai notamment des onomatopées, dont la sonorité est choisie dans le but de symboliser le concept visé. (Plouf!, par exemple, est censé reproduire le son d'un corps tombant dans l'eau.) Le signe linguistique est alors dit motivé.

Quoiqu'un peu plus complexe, le cas des idéophones s'apparente à celui des onomatopées. Par synecdoque, leur sonorité reflète explicitement une caractéristique du signifié pour désigner ce signifié lui-même. En lingala, par exemple, le mot niáo ([nao]), dont les caractéristiques phonétiques évoquent le miaulement du chat, est ainsi employé pour désigner l'animal' .

20 Contrairement à ce qui, selon Saussure, se produit sur le plan lexical, le signe linguistique est la plupart du temps motivé lorsque l'on se situe au niveau suprasegmental. Ceci est éminemment apparent lorsque l'on considère le cas de l'intonation, comme le souligne par exemple Liberman (1978) dans son étude consacrée

Corela, HS-29 | 2019 
à l'anglais américain : "A substantial part of the content of the intonational lexicon of English is determined by the universal symbolic (better: metaphoric) value of tones and tone-sequences." Certains principes intonatifs universels illustrent parfaitement cette motivation. Ainsi, dans la quasi-totalité des langues du monde, les contours descendants sont-ils associés à l'expression de la finalité, que celle-ci soit conçue de manière linguistique (grammaticale, pour signaler les énoncés assertifs, discursive, dans la régulation des tours de parole ${ }^{10}$ ) ou paralinguistique ${ }^{11}$, notamment attitudinale ${ }^{12}$. Par contraste, il est reconnu que les contours ascendants sont quasiuniversellement associés à l'expression de la non-finalité. Il est aisé de percevoir ici la motivation de l'intonation, qui opère par analogie avec le principe universel de la gravitation. Pour signaler qu'un propos reste en suspens, on utilise un schéma ascendant. De manière iconique, la voix reste pour ainsi dire suspendue dans les airs, et l'on s'attend à ce que le mouvement soit poursuivi par le locuteur lui-même ou ses protagonistes. À l'inverse, pour exprimer l'assertivité, on utilise un schéma descendant. La voix tombe jusqu'à atteindre son point final, signalant par là qu'il n'y a plus rien à attendre ou à ajouter.

\section{L'orientation des contours}

\section{Orientation descendante}

Dans notre corpus, la quasi-totalité des contours intonatifs observés sur les syllabes toniques sont orientés vers le bas (voir tableau 2 ainsi que les fig. 1 et 2, fournies à titre illustratif). Si tant est que cet échantillon de parole soit représentatif de la langue, l'exclamation semble donc, à l'instar des énoncés assertifs, être caractérisée par des contours descendants. Cela suggère que l'exclamation constitue une forme particulière d'assertion. Souvent, un énoncé exclamatif ne laisse en effet attendre aucune suite, ne sollicite aucune réponse. On pourrait compléter la définition de Quirk et al. (2004) proposée en introduction en suggérant qu'en s'exclamant, on asserte tout en signalant que l'on est impressionné ${ }^{13}$.

\begin{tabular}{|l|l|l|}
\hline & Descendant & Ascendant \\
\hline 1 & & \\
\hline 2 & & \\
\hline 3 & & \\
\hline 4 & & \\
\hline 5 & & \\
\hline 6 & & \\
\hline 7 & & \\
\hline 8 & & \\
\hline 9 & & \\
\hline
\end{tabular}




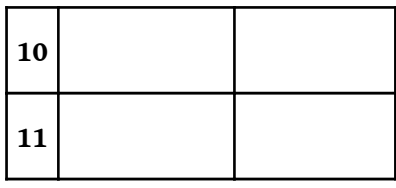

Tableau 2 : Orientation des schémas observés sur les toniques des énoncés du corpus

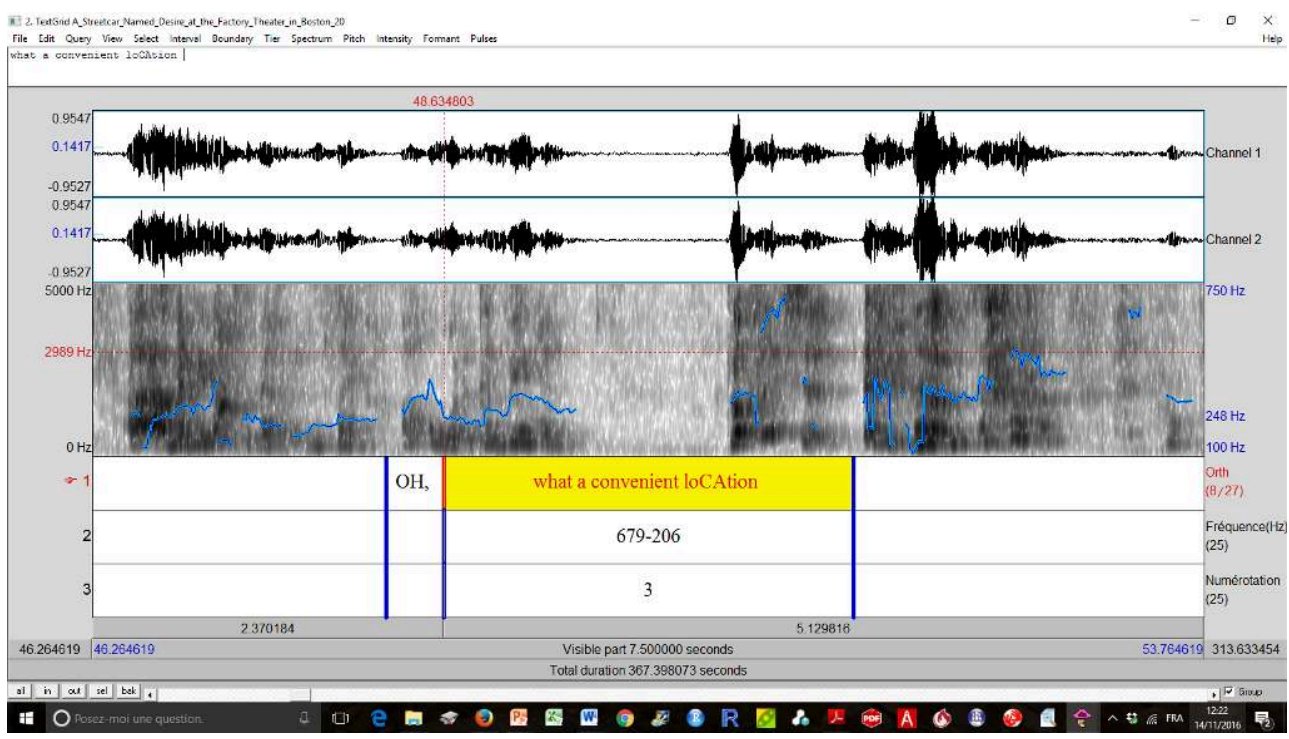

/ OH / what a convenient lollcation /

Fig. 1 : Spectrogramme et courbe de fréquence de l'énoncé $n^{\circ} 3^{14}$

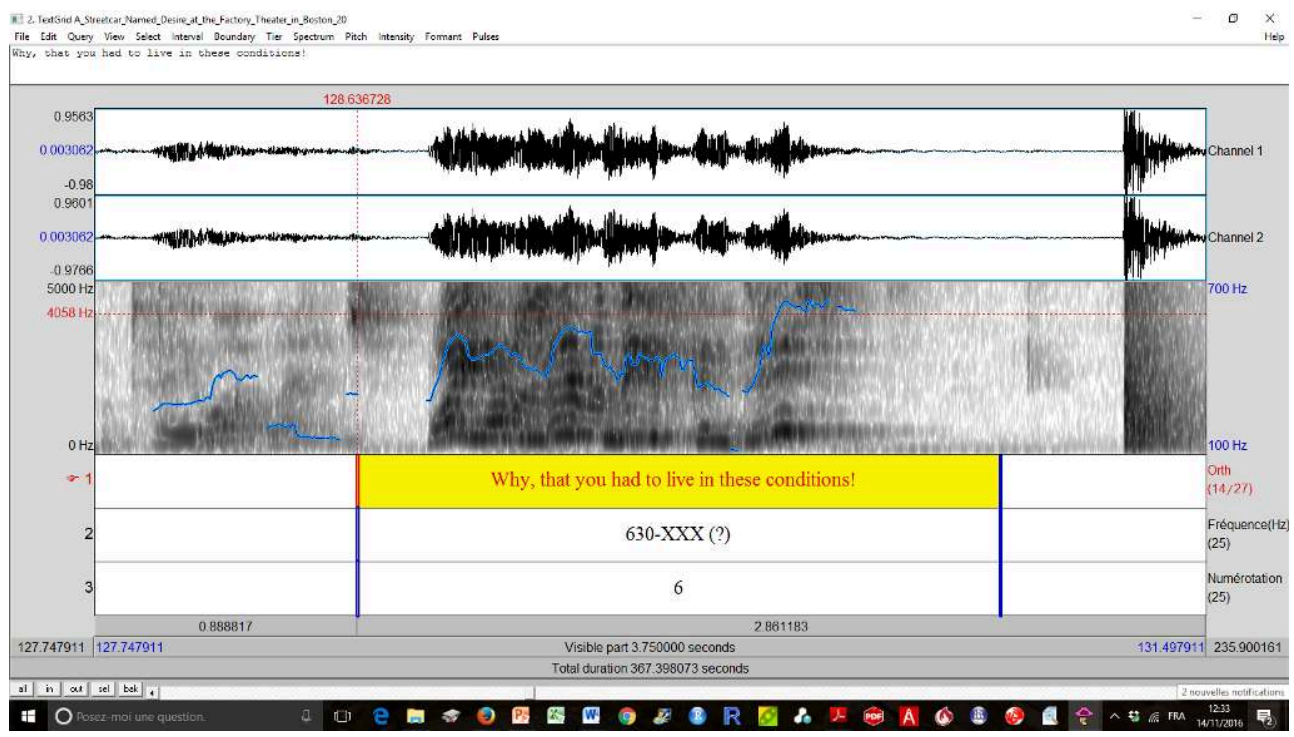

/ why, that you had to live in these con /

Fig. 2 : Spectrogramme et courbe de fréquence de l'énoncé $n^{\circ} 615$

\section{Orientation ascendante}

On observe pourtant dans le corpus deux énoncés dont l'intonation se trouve être ascendante. Il s'agit des occurrences $n^{\circ} 2$ (Turn that over-light off?) et $n^{\circ} 10$ (It's just incredible, Blanche, how well you're looking). 
l'énoncé $\mathrm{n}^{\circ} 2$, la présence d'un contour ascendant s'explique aisément par le fait que, dans l'interprétation de l'actrice, l'exclamation Turn that over-light off! n'est produite que comme une sorte de tremplin pour énoncer ce qui vient ultérieurement : Turn it off! D'un point de vue discursif, aucune finalité n'est par conséquent associée à Turn that over-light off! C'est la suite, dont le point de départ se situe plus haut dans la tessiture, qui est visée. On pourrait évoquer ici un phénomène de liaison intonative (voir figure 4). Ces considérations discursives prennent mécaniquement le pas sur l'iconicité de l'assertion.

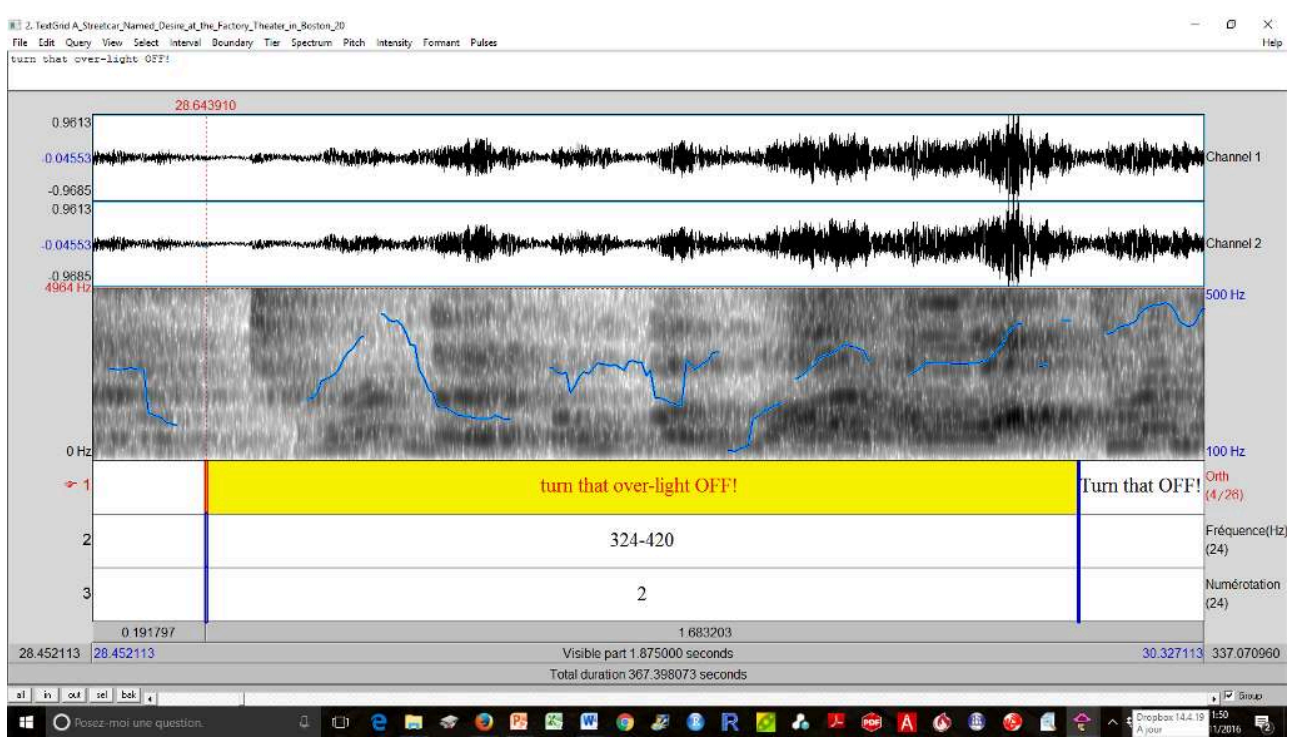

/ turn that over-light /off /

Fig. 3 : Spectrogramme et courbe de fréquence de l'énoncé $n^{\circ} 2$

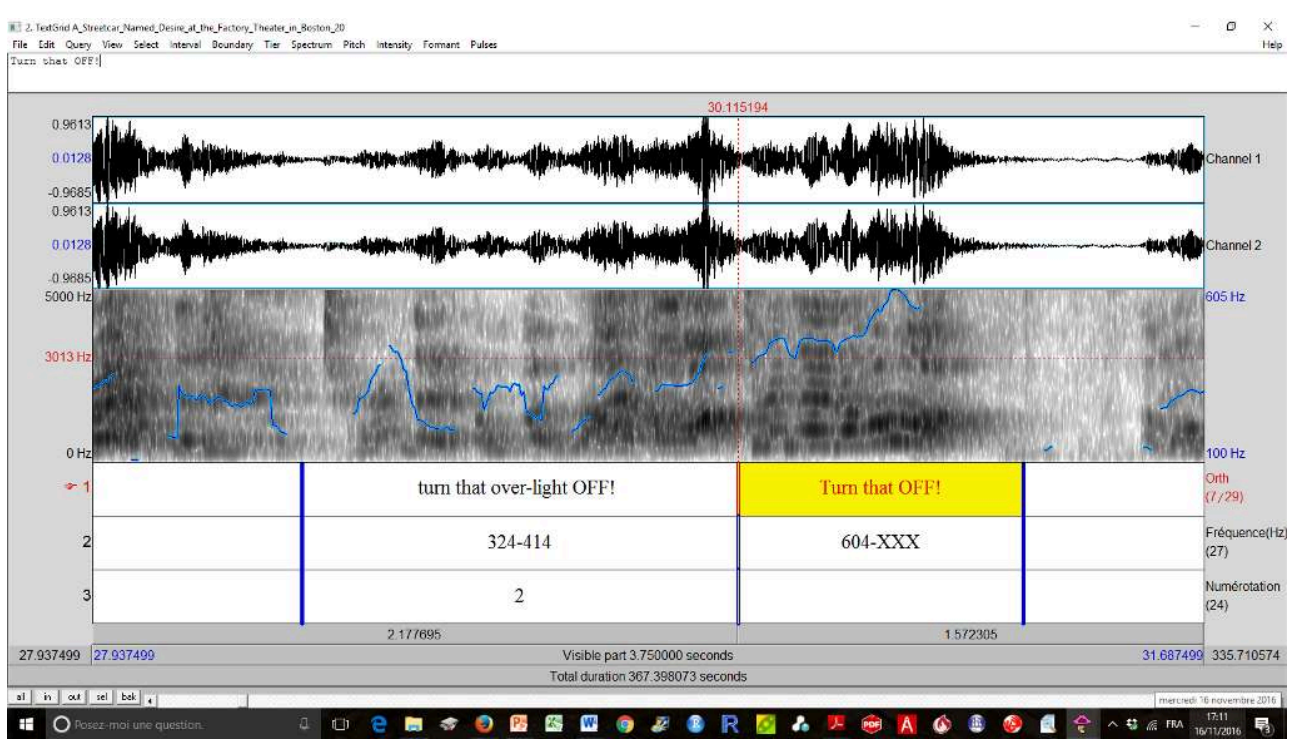

/ turn that over-light /off / turn it off /

Fig. 4 : SPECTROgRAMME ET COURBE de FRÉQUENCE de L'ÉNONCÉ N 2 ET DE SON COTEXTE DROIT ${ }^{16}$ 


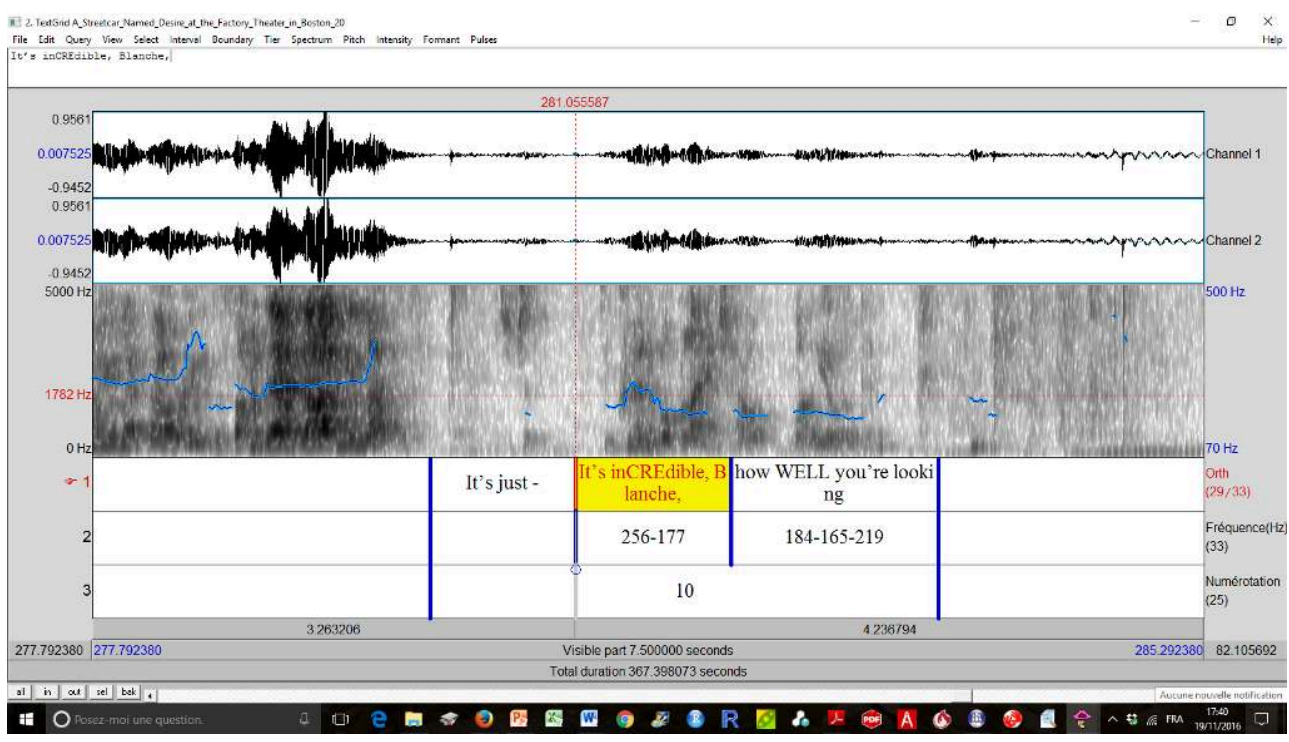

/ It's just in \credible, Blanche / how well you're (loo)king /

Fig. 5 : Spectrogramme et courbe de fréquence de l'énoncé $n^{\circ} 10$

Le cas de l'énoncé $\mathrm{n}^{\circ} 10$ (It's just incredible, Blanche, how well you're looking) est un peu plus complexe. Il s'agit d'une extraposition non prototypique (l'élément extraposé est une proposition en HOW), et le découpage de l'énoncé en unités intonatives s'avère malaisé. On pourrait en effet penser qu'il n'y a ici qu'une seule unité dont la tonique porterait un ton composé (voir par exemple Roach 2011). Notre avis est pourtant que nous sommes en présence de deux unités intonatives. Dans un cas d'extraposition classique, on n'aurait qu'une seule unité intonative. La tonique, qui porterait un ton descendant, serait vraisemblablement située sur l'adjectif, et la voix resterait dans le bas de la tessiture jusqu'à la fin de la seconde proposition. En l'occurrence, une première unité intonative est associée à la proposition en $\mathrm{IT}+\mathrm{BE}+\mathrm{adj}$. La tonique, qui tombe sur l'adjectif, porte un ton descendant. La seconde unité intonative correspond à la proposition extraposée. La tonique tombe sur l'adverbe well modifié par l'adverbe exclamatif HOW. Elle porte un ton complexe : après une légère chute mélodique, la queue du contour est ascendante.

À notre sens, cette configuration atypique s'explique par le fait que la fonction attitudinale de l'intonation se superpose ici à sa fonction linguistique et prime sur cette dernière ${ }^{17}$. Que l'on considère le ton sur lequel Stella s'exprime comme ironique ou que l'on se contente de voir dans sa réplique un mensonge de politesse proféré avec peu de conviction (malgré le contenu propositionnel [It's just incredible, Blanche, how well you're looking], Stella ne pense à aucun moment que sa sœur a bonne mine), on voit que l'attitude du locuteur signale un propos en suspens. À la proposition initiale, qui exprime la finalité, s'ajoute ainsi le signalement d'un manque d'assertivité. D'où l'orientation ascendante du schéma, malgré la présence du point d'exclamation à la fin de la seconde proposition. Notons que les choix intonatifs sont conditionnés par l'interprétation du script par les acteurs.

\section{Spécificité intonative de l'exclamation}

Le fait que la plupart des énoncés exclamatifs soient marqués par des schémas descendants ne saurait être considéré comme leur principale caractéristique 
phonologique distinctive, puisqu'il s'agit d'un trait qu'ils partagent avec l'ensemble des énoncés assertifs (voir la section traitant de la motivation de l'intonation). Comme le souligne Rett (2011), la spécificité prosodique essentielle des exclamations doit être définie autrement ${ }^{18}$. Beijer (2002), qui élabore sa réflexion à partir des travaux de Bolinger (1989), suggère que le trait symptomatique de l'intonation des exclamations réside dans le caractère "extrême" des contours qui les caractérisent: "What [...] exclamative intonation contours have in common is that they tend to reach for the extreme [...]". Ce caractère extrême peut résider dans l'amplitude remarquable du mouvement mélodique observé à partir de la tonique, mais aussi dans le fait que des mouvements relativement étroits soient observés à des niveaux extrêmes de la tessiture (Beijer 2002).

L'examen de notre corpus semble corroborer cette hypothèse, puisque la majorité des schémas qui y sont recensés (6 sur 11) comportent de telles caractéristiques (voir tableau 3).

\begin{tabular}{|c|c|c|}
\hline Énoncés & Caractéristiques extrêmes & Précisions \\
\hline 1 & & Mouvement à l'extrémité de la tessiture \\
\hline 2 & $\mathrm{X}$ & \\
\hline 3 & & Ampleur du mouvement mélodique \\
\hline 4 & & Ampleur du mouvement mélodique (interjection initiale) \\
\hline 5 & & Ampleur du mouvement mélodique (prédicat) \\
\hline 6 & & Ampleur du mouvement mélodique \\
\hline 7 & $\mathrm{X}$ & \\
\hline 8 & $\mathrm{X}$ & \\
\hline 9 & $\mathrm{x}$ & \\
\hline 10 & $\mathrm{x}$ & \\
\hline 11 & & Ampleur du mouvement mélodique (interjection initiale) \\
\hline
\end{tabular}

Tableau 3 : Présence de caractéristiques extrêmes dans les énoncés du corpus

Dans cinq cas sur six, les caractéristiques extrêmes de ces schémas intonatifs consistent en une ampleur marquée du mouvement mélodique. À titre illustratif, la fig. 1 montre la courbe de fréquence de l'énoncé $\mathrm{n}^{\circ} 3$ (Oh, what a convenient location), dont le contour est caractérisé par une chute mélodique de $473 \mathrm{~Hz}$, soit 20,64 demi-tons, ce qui correspond à près de deux octaves (!)

Dans l'énoncé $\mathrm{n}^{\circ} 1$ (Stella, Stella, Stella for star $!^{19}$ ), en revanche, l'amplitude du mouvement mélodique est minime $(73 \mathrm{~Hz}$, soit 2,21 demi-tons), mais le contour se trouve être réalisé à l'extrémité de la tessiture du locuteur, à une fréquence de plus de 
$600 \mathrm{~Hz}$ (voir fig. 6), alors que la ligne de base de la locutrice se situe en dessous de 200 $\mathrm{Hz}$

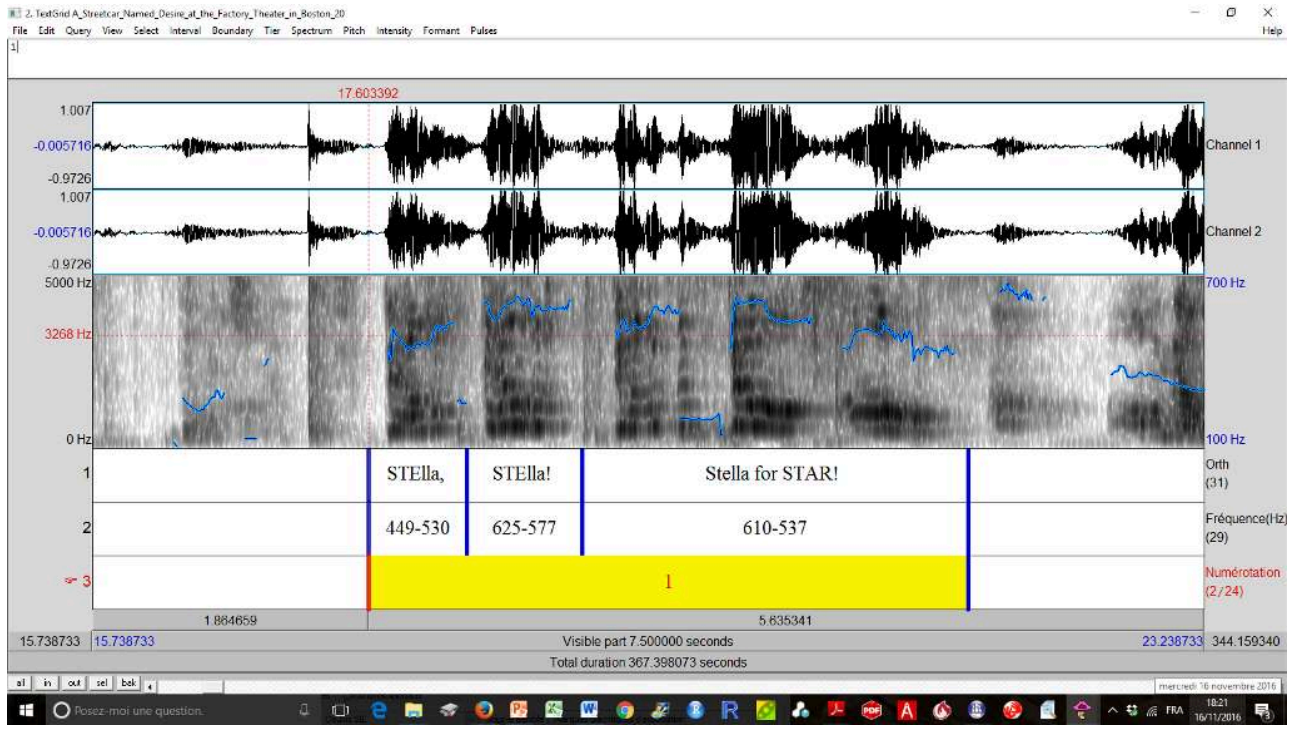

/ 'Stella / 'Stella / Stella for 'Star /

Fig. 6 : Spectrogramme et courbe de fréquence de l'énoncé $n^{\circ} 1$

31 Étant donné le caractère motivé et iconique de l'intonation, on comprend aisément que ces mouvements ou niveaux extrêmes puissent être employés pour représenter, en la mimant, l'intensité de l'émotion exprimée par le locuteur (cf. les définitions de Quirk et al. [2004] et de Bolinger [1989] fournies en introduction). On peut aussi envisager que ce caractère extrême résulte directement de l'émotion éprouvée au moment de leur production ${ }^{20}$. Ces deux explications ne sont pas incompatibles dans la mesure où, pour employer la terminologie de Jakobson, il n'est pas toujours aisé de circonscrire la fonction expressive et la fonction conative du langage.

\section{Synthèse provisoire}

A ce stade, notre examen du corpus suggère que dans les énoncés exclamatifs, l'intonation reste motivée et iconique, comme dans les autres types d'énoncés. À l'instar des énoncés assertifs prototypiques, l'exclamation est généralement exprimée par des schémas descendants traduisant la finalité. La pluralité fonctionnelle de l'intonation induit cependant parfois des variations, notamment lorsque des facteurs paralinguistiques, en particulier attitudinaux, se superposent aux critères linguistiques, c'est-à-dire grammaticaux ou discursifs. Le caractère distinctif de l'exclamation réside en le fait que l'intonation y mime - ou y traduit - l'intensité de l'émotion au moyen de schémas aux caractéristiques extrêmes, dont l'amplitude est particulièrement marquée ou que l'on observe à la limite de la tessiture. Dans ces conditions, il n'est pas étonnant que les contours les plus fréquemment associés à l'exclamation soient la grande chute et la chute extrême. 


\section{Intonation exclamative et expression du degré} signale l'expression du haut degré, le mot porteur de degré tel que l'entend Bolinger (1989) est l'adjectif becoming, qui désigne la propriété appréciée. À nouveau, c'est lui qui porte l'accent nucléaire.

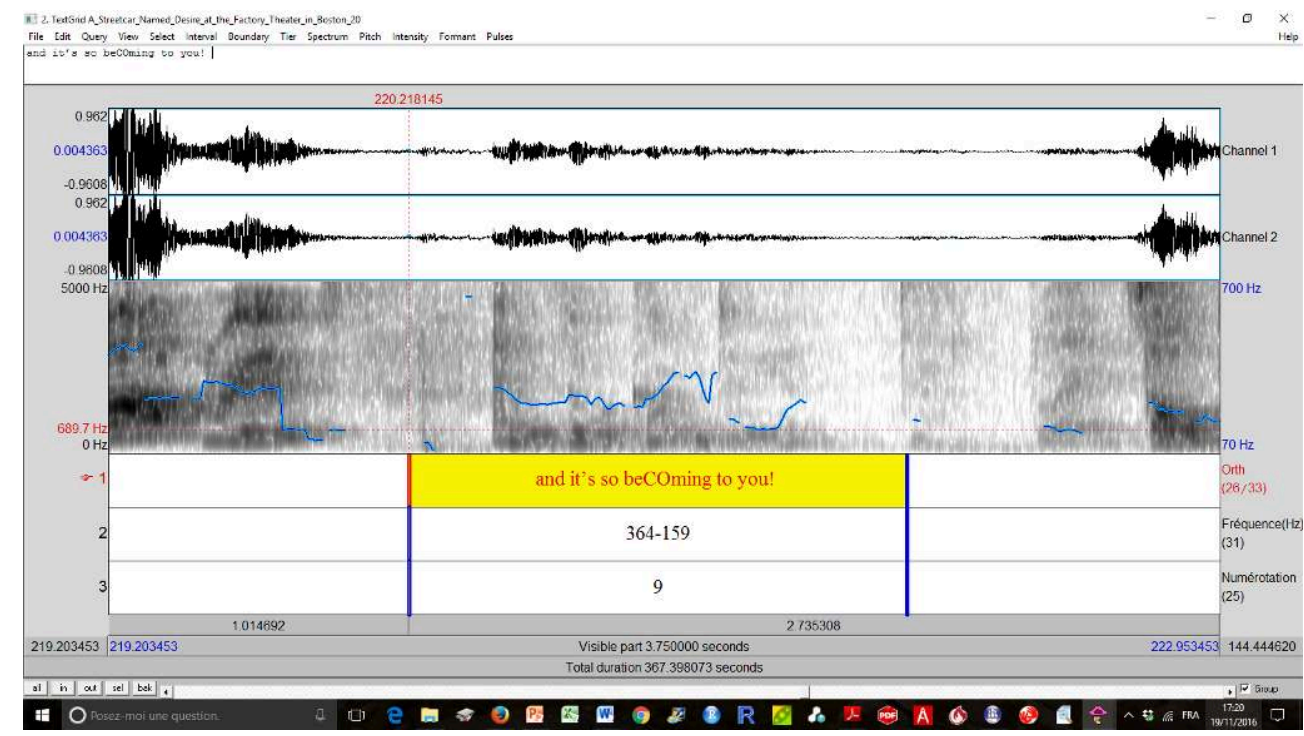

/ it's so belcoming to you /

Fig. 7 : Spectrogramme et courbe de fréquence de l'énoncé $n^{\circ} 9$

Se fondant sur les travaux de Bolinger, Beijer (2002) souligne qu'en contexte exclamatif, c'est en règle générale le mot qui exprime le degré qui porte l'accent de phrase.

According to Bolinger (1989) wh-exclamations, i.e. exclamations with initial whelements, almost universally have an intonation contour in which the degree-word has the (exclamatory) accent and much higher pitch than the rest of the utterance. In that way it is indicated that the degree-word is the most important word in the proposition. ne sont pas les marqueurs grammaticaux de degré tels que WHAT, SO, etc. que Bolinger désigne par les termes degree word ou degree expression, mais les mots lexicaux - verbes, substantifs, adjectifs - qui désignent une qualité présente à un degré qu'il est possible de mesurer sur une échelle (scale). prédéterminant WHAT est utilisé pour exprimer le haut degré, mais c'est l'ensemble convenient location qui désigne la notion dont les propriétés sont évaluées. Comme la plupart du temps dans ce type de suite adj. + N, c'est le nom qui porte l'accent principal. Dans cet énoncé, c'est par conséquent location qui porte l'accent de phrase.

On observe un phénomène comparable dans l'énoncé $n^{\circ} 9$ (It's so becoming to you!), dont forme syntaxique est celle d'un énoncé déclaratif en So. Si l'adverbe So, intensifieur,

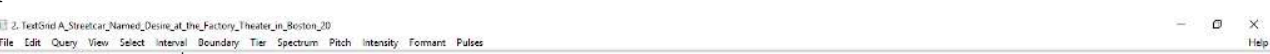

Un phénomène similaire est encore observé dans l'énoncé $n^{\circ} 6$ (why, that you had to live in these conditions!) (voir fig. 2). Dans ce cas précis, these peut être analysé comme un déterminant déictique utilisé pour construire une référence exophorique (il permet de désigner l'environnement dans lequel vit Stella tel que Blanche le perçoit), mais aussi comme un marqueur signalant le haut degré par boucle autoréférentielle. Une fois 
encore, le mot qui exprime les propriétés est le nom conditions (on a affaire à une forme d'euphémisme, ce substantif désignant implicitement des conditions évaluées comme misérables, etc.). C'est lui qui porte la tonique.

Puisque ce sont les mots qui expriment le degré qui portent l'accent de phrase dans les exclamations et que les contours esquissés à partir de la syllabe tonique y sont caractérisés par une large amplitude, il serait tentant de penser que les schémas intonatifs exclamatifs miment directement le haut degré des propriétés exprimées. La véritable fonction des énoncés exclamatifs est cependant de signaler l'émotion (cf. Quirk et al. 2004 et Bolinger 1989, cités en introduction). C'est par conséquent la réaction du locuteur qui est exprimée par l'exclamation et non le haut degré de ces propriétés en tant que tel. Par iconicité, l'amplitude extrême des contours renvoie à l'évaluation et à l'état intérieur de celui qui parle.

\section{Absence de haut degré}

On ne trouve aucun marqueur morphosyntaxique signalant le haut degré dans l'énoncé $\mathrm{n}^{\circ} 11$ (Yes, types is right!). Nul contour extrême n'est employé sur la syllabe tonique (voir fig. 8) et, sans la ponctuation qui figure dans le script, cette occurrence ne serait vraisemblablement pas même analysée comme une exclamation.

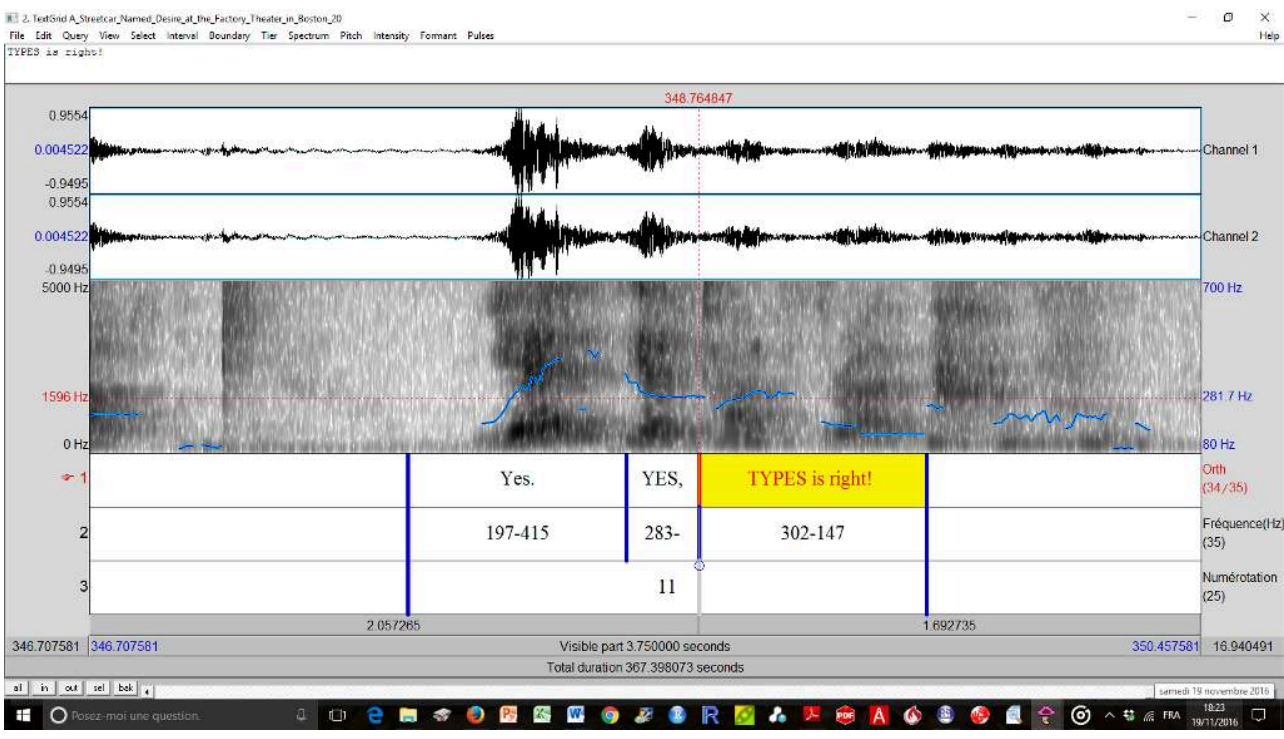

/ -yes / types is right /

Fig. 8: Spectrogramme et courbe de fréquence de l'énoncé $n^{\circ} 11$

Il se trouve en réalité un marqueur intonatif caractéristique dans la réalisation phonétique du contexte de l'énoncé, mais celui-ci n'est pas situé là où on l'attendrait. Bien que le point d'exclamation se trouve à la fin du segment Types is right!, c'est la première occurrence du mot Yes, produit deux unités intonatives avant l'énoncé $n^{\circ} 11$, qui se trouve réalisée assez haut dans la tessiture de l'actrice, signalant une émotion d'une relative intensité. On peut admettre que c'est bien l'énoncé Types is right! qui est visé : le contour employé sur le Yes initial est en effet ascendant, ce qui suggère que l'élément important est encore à venir.

41 Cette configuration inattendue s'explique entre autres par le fait que les propriétés exprimées par le mot types ne sont pas évaluées comme présentes à un haut degré. Stella se contente de reprendre le terme que Blanche a employé pour évoquer la 
personnalité des camarades polonais de son mari et confirme qu'à son sens, ce vocable est approprié. Il s'agit d'un simple choix entre deux valeurs ( $p$ et p', dans la terminologie de la Théorie des Opérations Énonciatives). En l'occurrence, c'est la valeur positive qui est retenue. C'est pourquoi l'actrice choisit de rendre le point d'exclamation en reportant le marqueur exclamatif de hauteur mélodique sur le Yes initial, marqueur d'acquiescement. Stella ne fait qu'attester qu'il y a bien correspondance entre la notion nommée par sa sœur et la réalité extralinguistique avec laquelle elle est familière.

Dans la mesure où l'intonation exclamative exprime l'émotion et non directement le degré des propriétés (voir supra), rien, en théorie, n'empêcherait qu'un choix entre des valeurs $p$ et $p$ ' ne déclenche une vive émotion et ne donne lieu à l'emploi d'un schéma intonatif aux caractéristiques extrêmes. Simplement, ici, cela n'est pas le cas ${ }^{21}$.

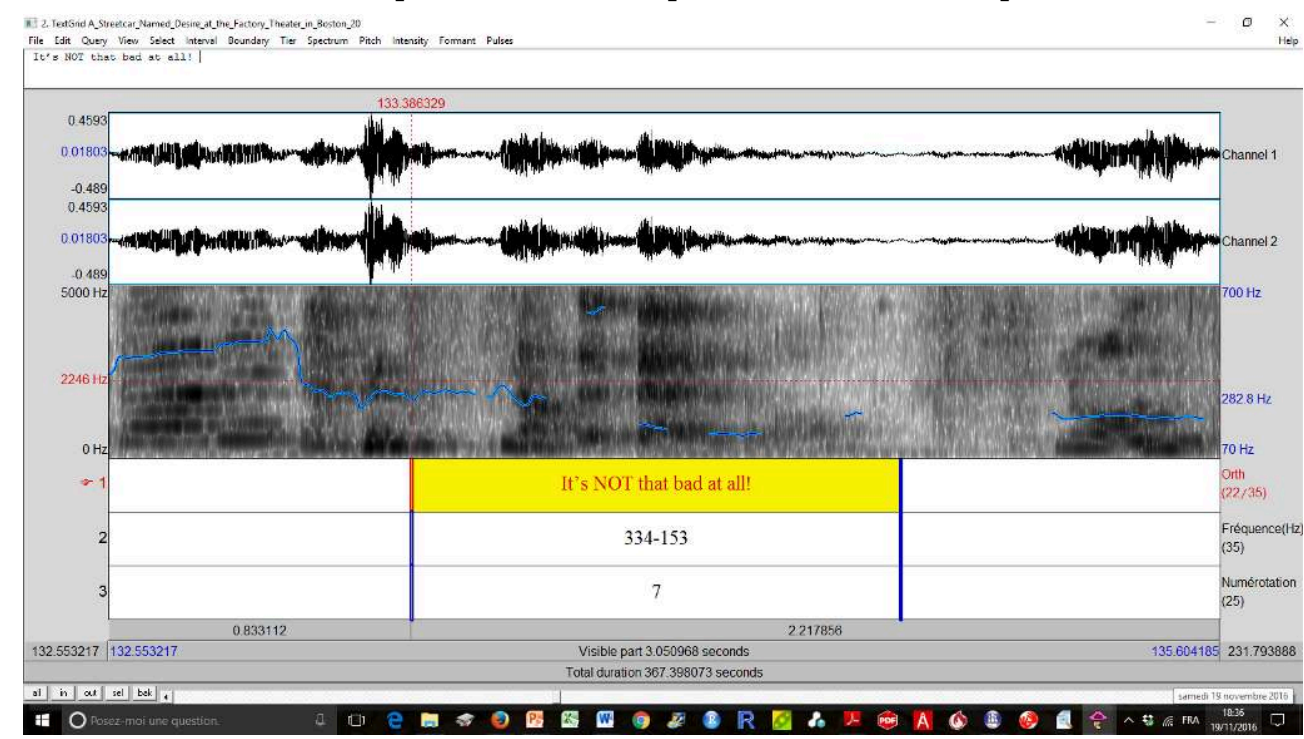

/ it's \not that bad at all /

Fig. 9 : Spectrogramme et courbe de fréquence de l'énoncé $n^{\circ} 7$

L'énoncé $\mathrm{n}^{\circ} 7$ (It's not that bad at all!) est comparable au précédent. On aurait pu imaginer que la tonique tomberait sur at all, locution qui intensifie la négation. Une chute extrême aurait alors été attendue pour rendre la valeur du point d'exclamation. $\mathrm{Au}$ lieu de cela, c'est le négateur not qui porte la tonique et se trouve réalisé avec une petite chute. À nouveau, l'absence de schéma extrême coïncide avec le fait que l'actrice met l'accent sur le choix entre deux valeurs possibles (that bad et not that bad) et non sur l'intensification. Si l'on considère la réalisation phonétique de l'énoncé en faisant abstraction du script, aucun élément n'évoque ici encore une exclamation. Faudrait-il parler dans ce cas précis de hiatus entre le texte de Tennessee Williams et l'interprétation qu'il reçoit ${ }^{22}$ ?

\section{Intonation, délimitation, énonciation}

On proposera dans cette dernière section une réflexion sur l'éclairage apporté par l'intonation des exclamations sur la définition même d'un acte d'énonciation.

Considérons tout d'abord à dessein une description non spécialisée de l'énoncé. Dans l' Encylopédie Universalis, on trouve la définition suivante : "Énoncé: séquence orale ou 
écrite résultant d'un acte d'énonciation, c'est-à-dire produite par un sujet énonciateur dans une situation donnée. » Rien n'est précisé ici quant à la nature de la séquence évoquée.

Pour certains, un énoncé prototypique consiste en une phrase syntaxique comportant un sujet et un prédicat. Dans notre corpus, on rencontre cette configuration dans les occurrences $\mathrm{n}^{\circ} 4$ (Oh, I spy, I spy!), $\mathrm{n}^{\circ} 7$ (It's not that bad at all!), $\mathrm{n}^{\circ} 9$ ([And] it's so becoming to you!) et $\mathrm{n}^{\circ} 11$ (Yes, types is right!). Mais il est largement reconnu que les énoncés peuvent aussi revêtir d'autres formes syntaxiques, plus complexes (c'est le cas de la construction extraposée dans l'exemple 9) ou moins élaborées (c'est vrai de l'énoncé 1 : Stella, Stella! Stella for Star!, où l'on observe un simple enchaînement de type SN / SN + $\mathrm{SP})$. Un énoncé peut parfois ne comporter qu'un seul $\operatorname{mot}^{23}$.

L'occurrence qui retiendra particulièrement notre attention est l'énoncé n 5 : Only Poe! Only Mr. Edgar Allan Poe!

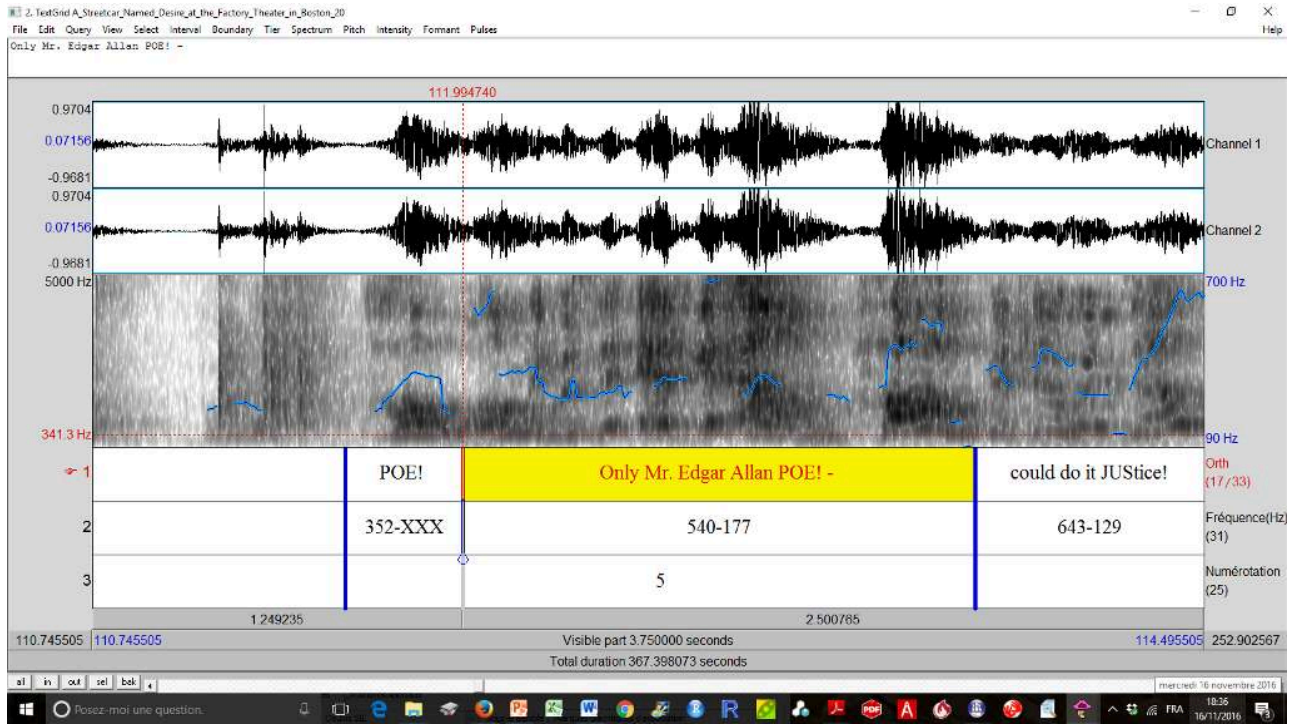

/ Poe / Only Mr. Edgar Allan \Poe /

Fig. 10 : Spectrogramme et courbe de fréquence de l'énoncé $n^{\circ} 5$

Ce qui est remarquable n'est pas tant qu'il s'agisse d'un simple syntagme nominal précédé d'un adverbe, puisqu'un énoncé peut ne comporter qu'un mot ou un syntagme unique, mais plutôt le fait que Mr. Edgar Allan Poe possède le statut de constituant d'un énoncé prototypique plus étendu. Il s'agit en effet du sujet syntaxique de la phrase Only Mr. Edgar Allan Poe could do it justice, dont le dernier mot se trouve suivi d'un point d'exclamation dans le script et porte un ton nucléaire descendant dans l'interprétation de l'actrice. (En l'occurrence, il s'agit d'une chute extrême réalisée sur la tonique de la seconde unité intonative, la première syllabe de justice [voir fig. 10]). 


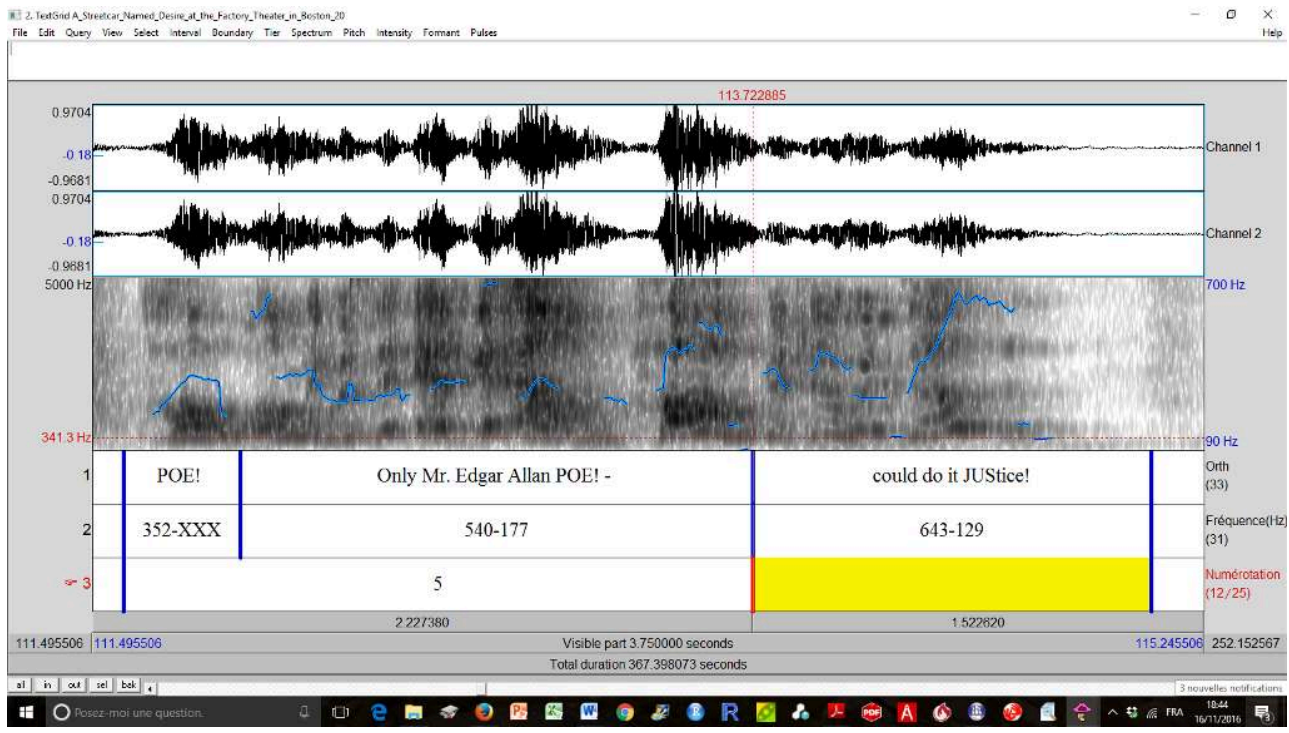

/ Poe / Only Mr. Edgar Allan \Poe / could do it /

Fig. 11 : Spectrogramme et courbe de fréquence de l'énoncé $n^{\circ} 5$ et de son cotexte

Mr. Edgar Allan Poe (tout comme Only Poe, juste avant l'occurrence soulignée) est bel et bien suivi d'un point d'exclamation dans le texte de Tennessee Williams. Faut-il alors considérer Only Mr. Edgar Allan Poe comme un énoncé à part entière? On hésite à apporter une réponse catégorique.

Quelques observations formulées en lien avec un autre phénomène intonatif, sans rapport direct avec les exclamations, sont susceptibles d'éclairer notre réflexion.

51 Les High Rising Terminals (HRT), largement répandus en anglais contemporain (Warren 2016), consistent en des schémas ascendants employés de manière non-canonique en fin d'énoncé déclaratif.

2 Le point crucial est ici le fait que les contours en question soient définis comme observés en fin d'énoncé (voir notamment Warren 2005, Fletcher \& Loakes 2006 et Wilhelm 2015). Dans la littérature - aujourd'hui relativement abondante --consacrée à ce phénomène, jamais cette définition n'a été remise en cause, même si certains auteurs préfèrent évoquer des schémas observés à la fin des "déclarations », des " affirmations » ou encore des "tournures déclaratives », des "phrases déclaratives » ou des « propositions déclaratives» (Wilhelm 2015).

De manière remarquable, il se trouve que l'examen de corpus oraux révèle la présence récurrente de tons phonétiquement et fonctionnellement similaires au High Rising Terminal à la fin de simples constituants d'énoncés prototypiques, voire de segments qui ne constituent pas même des constituants discrets (Wilhelm 2015). Cela soulève un problème d'ordre définitoire. Faut-il, en effet, postuler que ces tons ne sont pas à proprement parler des HRT? Et dans ce cas, comment expliquer leur frappante ressemblance avec ceux-ci ? Faut-il plutôt considérer que la définition du HRT doit être élargie et admettre que le High Rising Terminal puisse être observé sur des segments de discours qui ne doivent pas être considérés comme des énoncés ? Cette explication pose une sérieuse difficulté, car il devient alors malaisé de définir précisément ce qu'est un HRT. Faut-il enfin admettre que les constituants d'énoncés prototypiques sur lesquels les contours ascendants sont observés signalent des actes discrets d'énonciation ? Cette solution a le mérite de ne soulever aucune des épineuses difficultés posées par les deux 
autres hypothèses. Par analogie avec la terminologie de Brazil (1975; 1997), qui reconnaît la possibilité de conférer à un simple constituant une valeur " proclamative " (proclaiming) ${ }^{24}$, c'est la solution que nous proposons ailleurs (Wilhelm 2015).

Pour transposer cette analyse aux exclamations, il convient de dresser une rapide typologie des énoncés dont la force illocutoire est directement liée à l'expression de l'émotion. En nous inspirant librement des travaux de Beijer (2002) nous faisons l'hypothèse que ces énoncés sont susceptibles de revêtir trois types de valeurs :

55 - Certains ont une valeur expressive. Dans ce cas de figure, c'est avant tout le contenu propositionnel qui s'ajuste à l'émotion éprouvée ${ }^{25}$. Des énoncés comme $I$ am very surprised that she should have phoned ou I was appalled at the poor quality of the haggis réalisés avec une petite chute sur la tonique entreraient dans cette catégorie. - D'autres de ces énoncés peuvent avoir une valeur émotive. L'émotion n'est alors pas avant tout exprimée par le contenu propositionnel, mais également, voire exclusivement, par d'autres éléments - notamment la prosodie. Dans un énoncé comme That he should phone at this time of night! réalisé avec une chute extrême sur this, l'émotion serait par exemple exprimée par l'intonation et non par le contenu propositionnel.

57 - Les interjections constituent un cas à part, car elles traduisent une émotion sans qu'aucun contenu propositionnel ne soit exprimé. (C'est le cas dans des énoncés tels que Ah!, Aha!, Argh!, Oh!, Oops!, Wow!, etc.)

58 Ce que nous retiendrons est le fait qu'un contenu propositionnel n'est pas toujours nécessaire à la production d'un énoncé exclamatif. Ceci est particulièrement évident dans le cas des interjections, qui résultent d'actes d'énonciation discrets, ainsi, dans une moindre mesure, que dans celui des énoncés à valeur émotive, où le contenu propositionnel ne constitue pas l'élément qui confère à l'énoncé sa force illocutoire.

On conçoit donc qu'à l'instar des interjections, d'autres types de séquences dépourvues de contenu propositionnel soient susceptibles de constituer un énoncé lorsqu'elles sont marquées d'un point d'exclamation à l'écrit ou d'un schéma intonatif exclamatif à l'oral, indépendamment de leur structure syntaxique. On émettra par conséquent l'hypothèse qu'une exclamation signale invariablement un acte d'énonciation, même lorsqu'elle coïncide avec un simple constituant à l'intérieur d'un énoncé plus " étendu », comme c'est le cas dans l'exemple $n^{\circ} 5$ de notre corpus.

60 Cette manière de concevoir l'exclamation n'est pas sans soulever d'autres questions. Par exemple, quel statut faut-il reconnaître, dans ce cas, à ce qui « reste » de l'énoncé canonique dans lequel apparaît le « constituant exclamatif » une fois ce dernier analysé comme un énoncé à part entière ? En l'occurrence, comment faut-il analyser le prédicat could do it justice! une fois que Only Mr. Edgar Allan Poe! a été analysé comme résultant d'un acte discret d'énonciation? Dans le cas qui nous occupe, on peut en outre choisir de considérer que le schéma intonatif porte uniquement sur le groupe nominal $\mathrm{Mr}$ Edgar Allan Poe, dans quel cas l'intonation exclamative est bien observée en fin de constituant. Mais on pourrait aussi penser que le ton délimite l'ensemble de la séquence adv. + SN Only Mr Edgar Allan Poe. Dans ce cas, l'exclamation ne porterait pas même sur un constituant discret de l'énoncé plus étendu Only Mr. Edgar Allan Poe could do it justice! Notre propos n'est pas de poursuivre cette réflexion plus avant, mais on voit que l'intonation des exclamations amène à reconsidérer en profondeur des notions aussi fondamentales que celle de l'énoncé à l'oral. 


\section{Conclusion}

61 Les réflexions consignées ici soulèvent parfois autant d'interrogations qu'elles n'apportent de réponses, aussi est-il difficile de conclure. Nous nous contenterons ici de synthétiser les enseignements apportés par notre analyse du corpus et l'éclairage que celle-ci apporte sur la phonologie des exclamations.

Les caractéristiques phonologiques des énoncés exclamatifs - qui peuvent revêtir toute sorte de formes syntaxiques - doivent avant tout être examinées au niveau suprasegmental. La composante prosodique privilégiée pour exprimer l'exclamation est l'intonation, qui présente la particularité d'être motivée et iconique. Que la fonction des énoncés exclamatifs soit avant tout envisagée comme expressive ou conative, on trouve dans cette iconicité l'explication de la forme des contours qui y sont observés. De manière prototypique, l'exclamation est assertive et signalée par des schémas descendants reflétant la finalité. Elle est aussi et avant tout exprimée par des schémas aux caractéristiques extrêmes, qui traduisent l'intensité de l'émotion exprimée. C'est pourquoi les tons les plus typiques des énoncés exclamatifs sont la grande chute et la chute extrême, même si la pluralité fonctionnelle de l'intonation peut induire l'emploi d'autres contours.

Conformément à ce que souligne Bolinger (1989), dans les énoncés exclamatifs, l'accent de phrase marque fréquemment un terme qui exprime des propriétés présentes à un haut degré. Il convient toutefois de garder à l'esprit que le caractère extrême des contours employés ne reflète pas directement ce haut degré, mais l'émotion exprimée par le locuteur lorsqu'il est confronté à celui-ci.

Nous émettons enfin l'hypothèse que les exclamations signalent invariablement des actes discrets d'énonciation, même dans certaines configurations non-prototypiques, et en particulier lorsque les séquences syntaxiques qui leur correspondent n'expriment aucun contenu propositionnel. Ceci s'applique entre autres aux cas où des schémas exclamatifs délimitent de simples constituants d'énoncés prototypiques, voire des séquences qui ne consistent pas des constituants discrets de ces mêmes énoncés. Ces observations corroborent des hypothèses émises sur la nature des énoncés à l'oral en lien avec d'autres aspects de l'intonation de l'anglais. Elles constituent un domaine d'investigation qui gagnerait à être approfondi.

\section{BIBLIOGRAPHIE}

Beijer, F. (2002). “The syntax and pragmatics of exclamations and other expressive/emotional utterances". Working Papers in Linguistics 2, The Department of English in Lund.

Bolinger, D. (1989). Intonation and its Uses: Melody in Grammar and Discourse. Stanford: Stanford University Press.

Brazil, D. (1975). Discourse Intonation. Université de Birmingham : English Language Research. 
Brazil, D. (1997). The Communicative Value of Intonation in English. Cambridge : Cambridge University Press.

Cruttenden, A. (1995). "Rises in English", in W. J. LEWIS (éd.), Studies in General and English Phonetics: Essays in Honour of Professor J. D. O'Connor. Londres: Routledge.

Cruttenden, A. (1997 [1986]). Intonation ( $2^{\mathrm{e}}$ éd.). Cambridge : Cambridge University Press.

Halliday, M. A. K. (1967). Intonation and Grammar in British English. La Haye: Mouton.

Huddleston, R. \& Pullum, G. K. (2002). The Cambridge Grammar of the English Language. Cambridge: Cambridge University Press.

Jones, D. (1947 [1940]). An Outline of English Phonetics (6e éd.). Cambridge: Heffer.

Ladd, D. R. (2007 [1996]) Intonational phonology (Cambridge Studies in Linguistics 79) (2nd ed.).

Cambridge: Cambridge University Press.

Larreya, P. \& Rivière, C. (2002). Grammaire explicative de l'anglais. Londres: Longman.

Lehiste, I. (1970) Suprasegmentals. Cambridge, Massachussets: MIT Press.

Liberman, M. (1978). The intonational system of English. PhD. Dissertation, Massachusetts Institute of Technology.

O'Connor, J. \& Arnold, F. (1973). Intonation of Colloquial English. Bristol: Longman.

Quirk, R., Greenbaum, N., Leech, G. \& Svartvik, J. (2004 [1985]). A Comprehensive Grammar of the

English Language. Londres: Longman.

Rett, J. (2011). "Exclamatives, Degrees and Speech Acts". Proceedings of SALT 18.

Roach, P. (2011 [1983]) English Phonetics and Phonology (3rd ed.). Cambridge: Cambridge University Press.

Sweet, H. (1877). A Handbook of Phonetics. Londres: Clarendon.

Walker, J. (1974 [1787]). The Melody of Speaking. Londres : Scolar Press.

Wilhelm, S. (2011). Innovations segmentales et suprasegmentales dans le NW Yorkshire, Implications pour l'étude du changement accentuel dans l'anglais des îles Britanniques. Thèse de doctorat, Université de Bourgogne.

Wilhelm, S. (2015). «Quand les paroles s'envolent : réflexions sur les caractéristiques et la forme phonétique du High Rising Terminal en anglais contemporain ", Anglophonia, 20. URL: http:// anglophonia.revues.org/591

Warren, P. (2016). Uptalk. Cambridge: Cambridge University Press.

\section{NOTES}

1. "The concept of exclamation is a somewhat nebulous one." (Huddleston et Pullum 2002)

2. En l'occurrence, il n'est pas dénué d'intérêt de noter que tous les énoncés constituant le corpus comportent bien des points d'exclamations, excepté les occurrences $n^{\circ} 3$ et $n^{\circ} 10$, qui correspondent à des « constructions exclamatives » en WHAT et en HOW.

3. À l'instar de divers auteurs, nous considérerons les termes suprasegmental et prosodique comme synonymes. Le terme prosodie tire son origine du vocable grec prosōidia, qui désignait une pièce chantée avec un accompagnement musical. On peut en effet concevoir les éléments suprasegmentaux comme contribuant à la musicalité des énoncés dans la langue orale. 